\title{
ISP Traffic Engineering by Optimizing Routing Options: A Case of ZAMNET Communication Systems
}

\author{
Lazarous Tute ${ }^{1}$, Jackson Phiri ${ }^{2}$, Ariel Phiri ${ }^{3}$ \\ ${ }^{1,3}$ School of Engineering, Department of Electrical and Electronic Engineering, University of Zambia, Great East Road Campus, Lusaka \\ ${ }^{2}$ School of Natural Sciences, Department of Computer Studies, University of Zambia, Great East Road Campus, Lusaka
}

\begin{abstract}
As the number of Small to Medium Enterprises (SMEs) and individuals in Zambia accessing internet services today increases, there has been increased demand on service provider infrastructure. As the business trends have changed in communication, many organizations are turning to internet services for communication and sharing of resources. The SMEs also setup networks in order to make the process of sharing resources easier but riding on the Internet Service Provider (ISP) networks to interconnect their offices. The Internet Service providers have challenges providing internet services to the SMEs with consistent quality of service (QoS). There are limitations on either side, such as the provider network or client network, including high link congestion, intermediate node loss, high end-to- end delay and poor quality of service $(Q o S)$. These limitations lead to poor network performance which impact packet transmission and bandwidth provisioning to SMEs, as a result many complain of poor service. In this paper we present a study on the ISP network performance and the effect of changing the routing/switching options on service provisioning or delivery to the SME clients.
\end{abstract}

Keywords: Routed, switched network, packet delay, service plans.

\section{Introduction}

In Zambia internet services were introduced in the early 1990 s, with the use of e-mail only. This was after the formation of ZAMNET Communication Systems Limited as the first Internet Service Provider by the University of Zambia in 1994. In 1996, the then Communications Authority (CAZ), authorised ZAMNET to establish its own Very Small Aperture Terminal (VSAT) based data service. Data speeds achievable were very small typically between 14 -19 kbps. Over the years many Internet service providers have established their presence in Zambia, delivering different data services[1].

All over the world the Internet has become the main stay or platform for various networked services which have enhanced business including content distribution such as Voice Over Internet Protocol(VoIP), Internet Protocol Television (IPTV) and lately cloud computing. Given the popularity and the increasing reliance on these services, service failures and service quality degradations become ever costly in terms of reputation and revenue for service providers as well as for consumers. Thus, maintaining high service quality in the Internet has become increasingly important[2][3]. However, the Internet is a packet-switched, best-effort service based network. Unlike circuit-switched networks where on each path, dedicated resources are reserved end-to-end to guarantee service quality, there is no dedicated (virtual) circuit in the Internet for network level „,direct link ${ }^{\text {eee }}$ between two end systems, which is called a virtual path or simply path [4]. Because of this, the service quality of an Internet path highly depends on the underlying networking mechanisms, such as routing, buffer management and scheduling, plus the traffic condition on each hop along the path.
As a way of managing traffic in a network the concept of Traffic engineering becomes important. Traffic engineering (TE) is an important mechanism to optimize the performance of a data network by dynamically analyzing, predicting, and regulating the behavior of the transmitted data[5]. It has been widely exploited in the past and in current data networks, such as Asynchronous Transfer Mode (ATM) and Internet Protocol/Multi-Protocol label Switching (IP/MPLS) networks. Internet applications require the underlying network architecture to react in real time and to be scalable for a large amount of traffic[6]. The architecture should be able to classify a variety of traffic types from different applications, and to provide a suitable and specific service for each traffic type in a very short time period. Facing the rapid growth in cloud computing and thus the demand of massive-scale data centers, a fitting network management should be able to improve resource utilization for better system performance [7].

According to the Cisco Visual Networking Index, today video traffic contributes already more than $33 \%$ of the total consumer Internet traffic and this fraction was predicted to grow to $91 \%$ by 2014 . Video applications constitute a major challenge for content providers and network operators since they require a high amount of network resources in order to deliver a good experience[8].

Presently, Multiprotocol Label Switching (MPLS) is known to be the most effective at managing traffic and quality of service[5],[9]. MPLS can be used from edge-to-edge in a converged data and voice network. Furthermore MPLS performs across a variety of physical layers to enable efficient data forwarding together with reservation of bandwidth for traffic flows with different Quality of Service (QoS) requirements. The ability to provide service 


\section{International Journal of Science and Research (IJSR) \\ ISSN (Online): 2319-7064}

Index Copernicus Value (2013): 6.14 | Impact Factor (2014): 5.611

differentiation and resource assurance in a network is referred to as Quality of service. Resource assurance in information systems is based on per flow resource reservation meaning that before an application can transmit data into the network, it must make a resource reservation which involves several steps. Resource reservation makes sense for long lasting connections, but it is not appropriate for short lived connections like http traffic.

In addition, MPLS can operate on top of various routing protocols, including Open Shortest Path (OSPF), Routing Information Protocol (RIP) and Border Gateway Protocol (BGP)[10],[11],[12].

The demands placed on the internet continue to increase with the increased development of multimedia and other bandwidth intensive applications. It is thus necessary to extend the current best effort internet to support QoS to satisfy the end-to-end performance requirements of these applications. Possible setups or architectures that support QoS are recognized as an important step for the next generation internet service offerings[13].

On the other hand the internet is a commercial environment with different players or service providers competing with each other to provide services to a variety of end users by provisioning varied end-to-end (e2e) quality of service contracts with the flexibility of single-domain edge-to-edge (g2g) contracting capabilities. Current single domain contracts or service level agreements (SLAs) are typically point-to-anywhere settlements happening in peer-to-peer or customer provider ISP relationships[3][14].

In Zambia like most third world countries, Telecommunications infrastructure development has lagged behind though some inroads have been made now. In general Internet Service provisioning is dependent on available Telecommunications Infrastructure and therefore many of the Internet Service providers have had to deploy new networks to enhance performance and delivery of service at great cost. Where a Provider is not able to invest in infrastructure, there are options to use infrastructure sharing. In terms of service delivery,there are a variety of high speed Broadband internet access packages on the market, with different pricing strategies ranging from usage based to fixed monthly internet charges that users can choose from. The market is highly unregulated and lacks uniform pricing[1]. Effective competition plays a key role in expanding broadband access and in ensuring that consumers benefit from lower prices, greater choice and better quality of services.

In the European Union (EU), the EU Commission defined broadband internet service as "an access option assuring an always-on service with speeds in excess of $144 \mathrm{kbps}$, this speed is measured in download terms. During the nineties, broadband was delivered over cable and Telephone lines, in the years that followed, these technologies were upgraded and some operators began to deploy fibre for home delivery as that would support higher bandwidth[15].Also there has been an increase in the use of wireless technologies.

In comparison to Canada, the Canadian Federal and
Provincial Governments defined broadband as a connection that provides a minimum of download speed of $1.5 \mathrm{Mbps}$. This level of performance may be sufficient for first Generation internet applications such as e-mail and simple browsing ,but is not sufficient for deploying more advanced applications such as multimedia, Internet Protocol Television (IPTV) and Cloud Computing services[16].

In terms of pricing, it is seen that countries that use bit stream access have higher pricing than the ones that use direct access (local loop unbundling), as for Telecommunications operators they adapt their pricing and commercial strategies according to market evolution and competition[15].

\section{Literature Review}

The demands placed on internet continue to increase with the increased development of multimedia and other bandwidth intensive applications. It is thus necessary to extend the current best-effort internet to support Quality of service to satisfy the end-to-end performance requirements of these applications. Possible setups or architectures that support QoS are recognized as an important step for the next generation internet service offerings[13].

Broadband access can be provided via several Technologies as earlier alluded to such as XDSL, cable modem, FTTX or Wireless Technologies such as 3G,WiMax and Satellite [17].Competition in the retail Broadband services also depends on effective competition at the wholesale level or if this does not exist, on its regulation[18].

In recent years prices of fixed broadband internet access have fallen significantly. The prices of the service plans offered may vary according to the quality of the service and the Access Technology. It is also observed that Operators can release a large number of service plans to generate some confusion among consumers. The bandwidth or speed of the service is an important feature usually considered by consumers when they contract a service plan because it determines how fast they can view web pages, receive emails or download music.[15]

While Resource assurance defines a required service level, Resource allocation performs the task of sharing or allocating the available link resources in accordance with the assured service levels. One way of managing traffic in a network is the creation of Virtual Local Area Networks (VLANs).VLANS rely on Ethernet ability to carry traffic in defined and separated networks riding on the shared media, but the standardized QoS mechanism are limited, notably the IEEE $802.1 \mathrm{q}$ protocol only provides a mechanism for tagging Ethernet frames so as to indicate the deserved QoS characteristics[19]. An alternative QoS could be realized by means of IP-Protocol based instruments like Differentiated Services (DiffServe, a simple protocol that signals the desired QoS) and or MPLS (Multi-protocol label switching) or conceivably by Resource Reservation Protocol (RSVP, the rarely implemented protocol of the integrated service Architecture). Diffserve and MPLS cannot guarantee a

\section{Volume 5 Issue 1, January 2016}




\section{International Journal of Science and Research (IJSR) \\ ISSN (Online): 2319-7064}

Index Copernicus Value (2013): 6.14 | Impact Factor (2014): 5.611

connection with dedicated QoS features like ATM, but they can provide probabilistic assurance[20].

It is also important to note that internal routing/switching inside the network of an Internet Service Provider affects the performance of lots of services that the ISP offers to its customers and it is therefore critical to adhere to service level Agreements(SLA) to achieve a top quality offer and earn revenue.[21]

As a consequence, the access methods at the client end, does affect the performance of the network. Some wireless/radio Technologies suffer from media access symmetry, especially Technologies using a central Base station and several mobile or fixed clients as this requires a ready to send/clear to send protocols (RTC/CTS) with a large turnaround time caused by the radio. The MAC protocol makes it more expensive to switch the direction of transmission than to transmit in one direction continuously. The drop rate is also significantly higher in wireless networks, thus affecting quality of service.

\section{Methodology}

\subsection{Initial Study}

In this study we carried out measurements on live data from ZAMNET network (the internet service provider) to collect the baseline performance using NAGIOS tool and ETR Bandwidth manager and examined the clients based on the service plans for the service delivery. The service plans constitute the levels of service defined based on the bandwidth available on that service plans. The range of Shared service plans starts from Home (Home based users), SU (Single Business Users), SOHO (Small office network), SME (Small to Medium Enterprise) and ME (Medium Enterprise).The service plans are also indicative of the achievable speeds when downloading or uploading content.

In capturing traffic the tool captures data based on the traffic flows either http or https or by port type, different other traffic patterns are captured as well. A typical capture appears as shown in figure 2. During the duration of the observation, the data was captured at different time frames. In this study the usage per service plan measured in Gigabits was used to evaluate the performance of the service .In Figure 2, is a typical capture of traffic going through a client link, measured over a one month period.
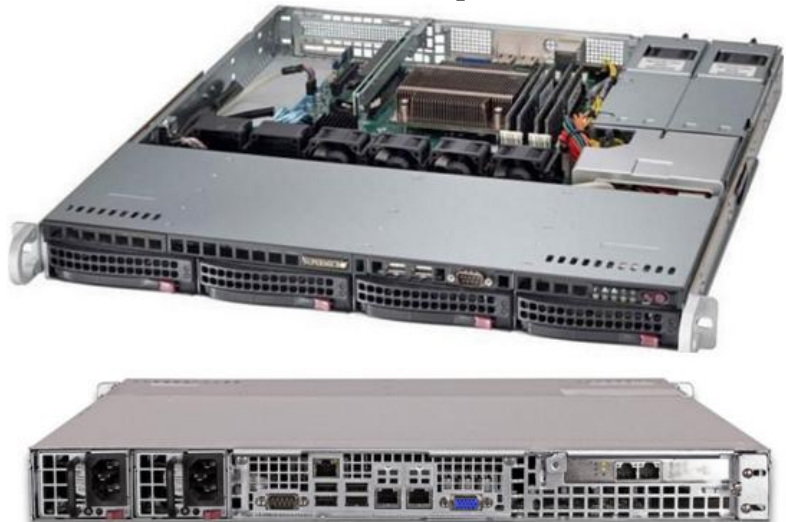

Figure 1: ETR 2800 Bandwidth Manager Device
The ETR 2800 devices allow the creation of service plans based on the proposed speed for that particular service plan. For instance, one service plan can be called home, with possible speed of $192 \mathrm{kbps}$ on a shared service plan.

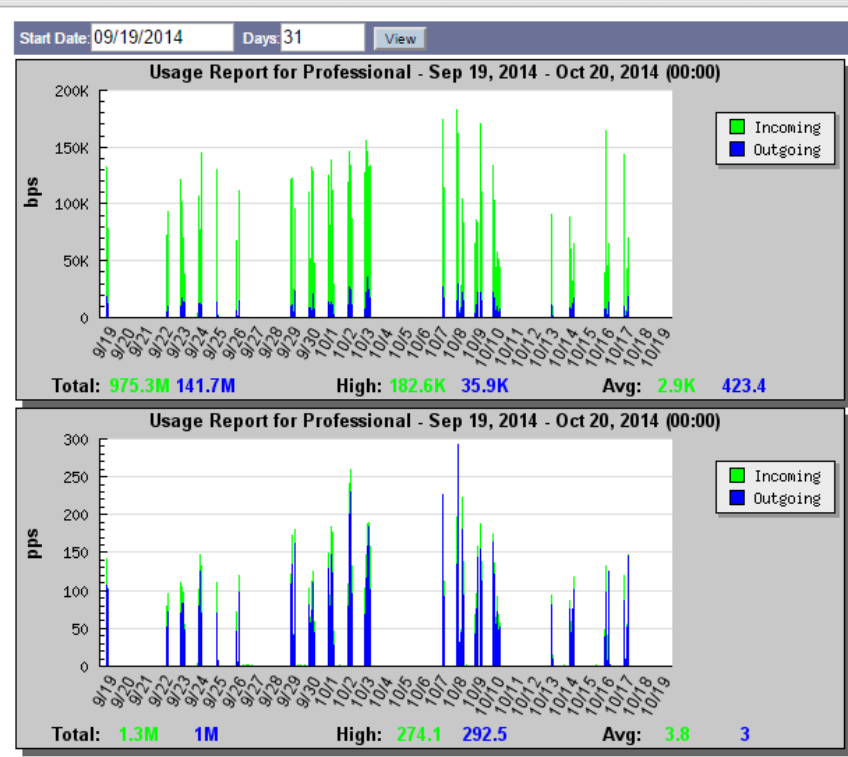

Figure 2: Capture on Data recorded in a month on ETR 2800 Device

Another tool called TTCP was used to capture data on the active links. TTCP is a utility that calculates the approximate through put in kilobytes per second between two selected nodes, it is a utility implemented in CISCO IOS 11.2 and later. To use TTCP , the utility is started in receive mode at one place within the network, then a second copy is started in transmit mode at another place within the network. The results of the transfer of data from the transmitter to the receiver indicate the approximate performance of the path between the source and destination. By selecting the source and destination at various points within the network, critical points of the path can be analyzed. TTCP uses the time and the amount of Data transferred to calculate the network performance.

A session on TTCP was setup to capture some performance details. Two routers are selected on the network.

\section{Host_one\#ttcp}

transmit or receive [receive]: transmit

Target IP address: 10.10.206.1

perform tcp half close [n]:

send buflen [8192]:

send nbuf [2048]:

bufalign [16384]:

bufoffset [0]:

port [5001]:

sinkmode $[\mathrm{y}]$ :

buffering on writes [y]:

show tcp information at end $[\mathrm{n}]$

ttcp-t: buflen $=8192$, nbuf $=2048$, align $=16384 / 0$, port $=5001$ tcp $->10.10 .206 .1$

ttcp-t: connect 


\section{International Journal of Science and Research (IJSR) \\ ISSN (Online): 2319-7064}

Index Copernicus Value (2013): 6.14 | Impact Factor (2014): 5.611

ttcp-t: 16777216 bytes in $12768 \mathrm{~ms}$ (12.768 real seconds) $(\sim 1283 \mathrm{kB} / \mathrm{s})+++$

ttcp-t: $2048 \mathrm{I} / \mathrm{O}$ calls

ttcp-t: 0 sleeps $(0 \mathrm{~ms}$ total) $(0 \mathrm{~ms}$ average $)$

\section{Host_two\#ttcp}

transmit or receive [receive]: receive

perform tcp half close [n]:

receive buflen [8192]:

bufalign [16384]:

bufoffset [0]:

port [5001]:

sinkmode $[\mathrm{y}]: \mathrm{y}$

rcvwndsize [4128]:

delayed ACK [y]:

show tcp information at end $[\mathrm{n}]: \mathrm{y}$

ttcp-r: buflen $=8192$, align $=16384 / 0$, port $=5001$

rcvwndsize $=4128$, delayed ack $=$ yes tcp

ttcp-r: accept from 10.10.207.1 (mss 536, sndwnd 4128, revwnd 4128)

ttcp-r: 16777216 bytes in $12772 \mathrm{~ms}$ (12.772 real seconds) $(\sim 1282 \mathrm{kB} / \mathrm{s})+++$

ttcp-r: $4149 \mathrm{I} / \mathrm{O}$ calls

ttcp-r: 0 sleeps ( 0 ms total) $(0 \mathrm{~ms}$ average $)$

Connection state is CLOSEWAIT, I/O status: 7,Connection is ECN Disabled, Mininum incoming TTL 0, Outgoing TTL 255

Local host: 10.10.206.1, Local port: 5001

Foreign host: 10.10.207.1, Foreign port: 59071

iss: 1386485913 snduna: 1386485914 sndnxt: 1386485914 sndwnd: 4128

irs: 1147388614 revnxt: 1164165832 revwnd:

3280

delrcvwnd: 848

Datagrams (max data segment is 536 bytes):

Rcvd: 32866 (out of order: 0), with data: 32863, total data bytes: 16777216

Sent: 30454 (retransmit: 0 , fastretransmit: 0 , partialack: 0 , Second Congestion: $\quad 0$ ), with data: 0 , total data bytes: 0

Overall various approaches to measuring network performance have been developed to date. At the end-user level, there are many utilities that allow even novice users to monitor speeds and other characteristics of their internet connections. However aggregating individual tests into indicators of performance reflective of larger systems is difficult and controversial. As a result there is little consensus about which approach to measuring speed captures reality in absolute terms. Furthermore the market for internet connectivity is highly differentiated in terms of both supply and demand. As a result significant caution must be used in interpreting aggregate indictors of Broadband network outcomes (e.g. Availability, price, speed etc.). Two basic approaches to collecting speed measurements have been developed and used in policy discussions.

Active Tests require an action by end-users to initiate a process that assesses the quality of internet connectivity e.g. download/upload speeds (latency, jitter etc.)

Passive Tests compile performance measurements without an explicit decision by end users to initiate a test, usually during the content application, delivery process measurements from Akamai, Google, YouTube and Netflix represent examples of the Passive approach[22][23].

\subsection{Network Setup}

To improve the network performance we designed and implemented a switched network using layer 3 VLANS on one segment of the network covering segments $\mathrm{A}$ and $\mathrm{C}$. The segment covering B was left with the original configurations. Data was collected for a period of two months and then analyzed.

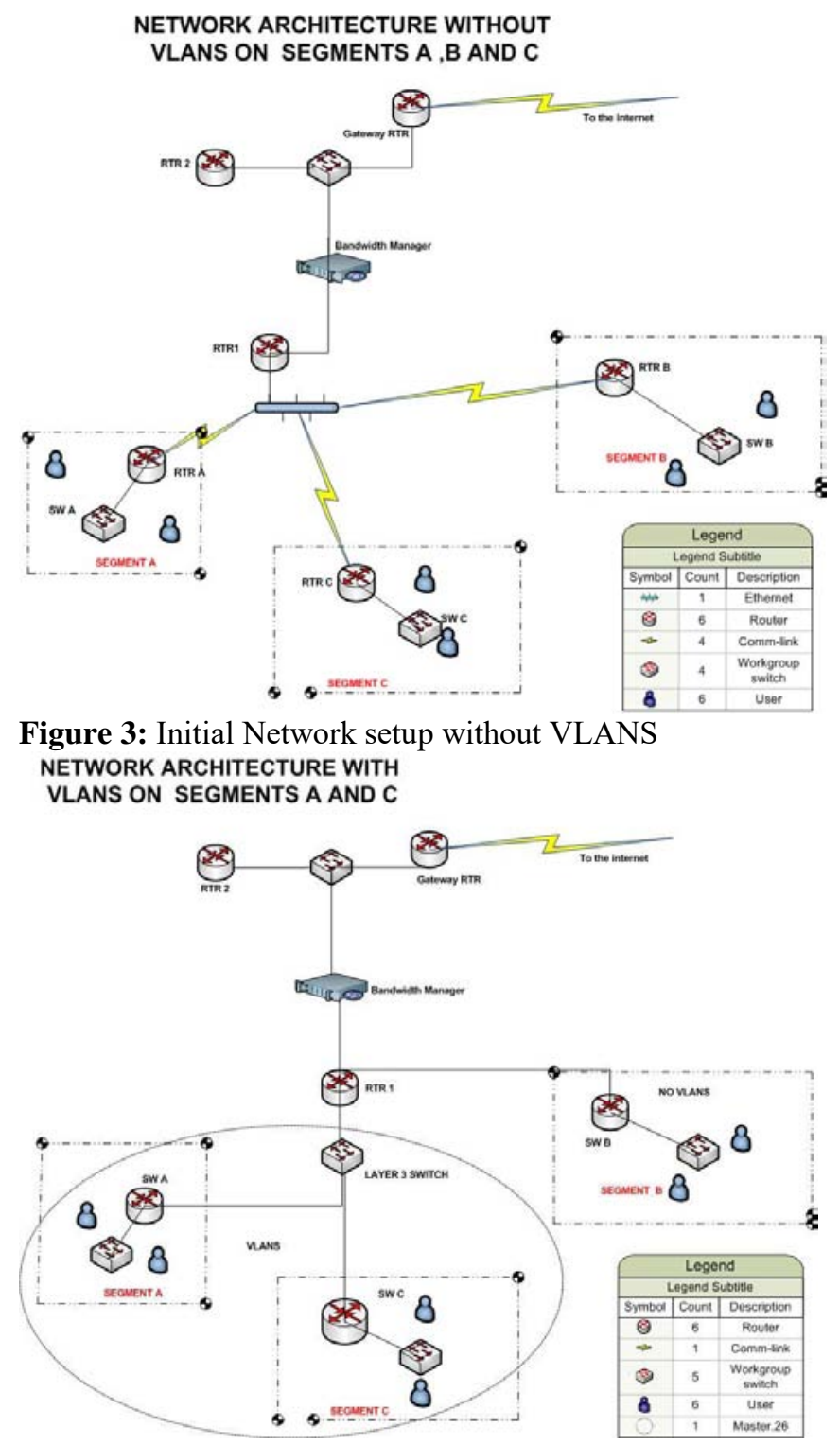

Figure 4: Network setup with VLANS

\subsection{Results and Discussion}

The data captured for the service plans in terms of usage are shown in the following tables set as Home(Home based users), Single Business User(SU),SOHO ( Small office network),SME( Small to Medium Enterprise),ME (Medium Enterprise). Figure 5, shows the data captured during the baseline study with Figure 6, indicating the graphical representation after the network was modified. Further data was collected for a period of two months and presented in graphical form as well. 


\section{International Journal of Science and Research (IJSR) \\ ISSN (Online): 2319-7064}

Index Copernicus Value (2013): 6.14 | Impact Factor (2014): 5.611

Table 1: Data Collection before modification of network

\begin{tabular}{|c|c|c|c|}
\hline Service Plan & $\begin{array}{c}\text { Incoming } \\
\text { Usage GB }\end{array}$ & $\begin{array}{c}\text { Outgoing } \\
\text { Usage GB }\end{array}$ & $\begin{array}{c}\text { Combined } \\
\text { Usage GB }\end{array}$ \\
\hline All_home_Total & 488.47 & 137.68 & 626.15 \\
\hline All_SU_Total & 425.12 & 107.33 & 532.45 \\
\hline All_SOHO Average & 342.24 & 97.95 & 440.19 \\
\hline All_Average_SME & 324.65 & 116.38 & 441.03 \\
\hline All_ME & 365.88 & 81.95 & 447.83 \\
\hline All ded_512 & 377.07 & 89.31 & 466.39 \\
\hline
\end{tabular}

\section{Usage per service plan -Month1}

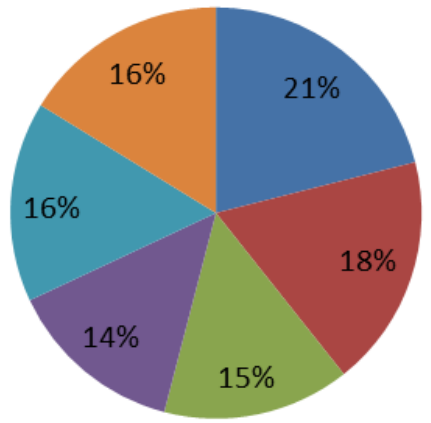

$$
\begin{aligned}
& \text { all_home_Total } \\
& \text { All_SU_Average } \\
& \text { All_SOHO Average } \\
& \text { all_Average_SME } \\
& \text { All_ME } \\
& \text { All ded_512 }
\end{aligned}
$$

Figure 5: Graphical Representation for 1st Month

Table 2: First Month after modification of network

\begin{tabular}{|c|c|c|c|}
\hline Service Plan & $\begin{array}{c}\text { Incoming } \\
\text { Usage GB }\end{array}$ & $\begin{array}{c}\text { Outgoing } \\
\text { Usage GB }\end{array}$ & $\begin{array}{c}\text { Combined } \\
\text { Usage GB }\end{array}$ \\
\hline All_home_Total & 1930.96 & 518.8 & 2449.75 \\
\hline All_SU_Average & 1603.45 & 370.08 & 1973.54 \\
\hline All_SOHO Average & 1004.22 & 250.22 & 1254.44 \\
\hline All_Average_SME & 1283.6 & 839.26 & 2122.86 \\
\hline All_ME & 1161.21 & 258.23 & 1419.43 \\
\hline All ded_512 & 568.35 & 151.03 & 719.4 \\
\hline
\end{tabular}

\section{Usage per service plan -Month2}

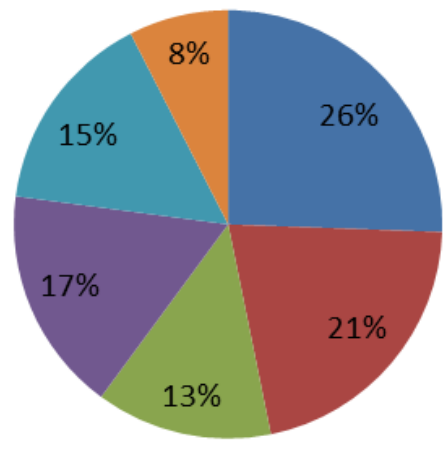

- All_home_Total

口 All_SU_Average

- All_SOHO Average

- All_Average_SME

- All_ME

all ded_512

Figure 6: Graphical Representation of First Month Data

Table 3: Second month after modification of network

\begin{tabular}{|c|c|c|c|}
\hline & $\begin{array}{c}\text { Incoming } \\
\text { Usage GB }\end{array}$ & $\begin{array}{c}\text { Outgoing } \\
\text { Usage GB }\end{array}$ & $\begin{array}{c}\text { Combined } \\
\text { Usage GB }\end{array}$ \\
\hline All_home_Total & $\mathbf{1 3 1 2 . 9 2}$ & $\mathbf{3 3 8 . 5 4}$ & $\mathbf{1 6 5 1 . 4 9}$ \\
\hline All_SU_Average & $\mathbf{9 7 6 . 8 2}$ & $\mathbf{4 1 4 . 0 3}$ & $\mathbf{1 3 9 0 . 8 4}$ \\
\hline All_SOHO Average & $\mathbf{8 6 2 . 6 4}$ & $\mathbf{2 8 0 . 6 1}$ & $\mathbf{1 1 4 3 . 2 5}$ \\
\hline All_Average_SME & $\mathbf{6 4 2 . 5}$ & $\mathbf{1 5 0 . 6 6}$ & $\mathbf{7 9 3 . 1 8}$ \\
\hline All_ME & $\mathbf{9 9 6 . 0 3}$ & $\mathbf{2 0 0 . 5 9}$ & $\mathbf{1 1 9 6 . 6 2}$ \\
\hline All ded_512 & $\mathbf{5 5 0 . 2 6}$ & $\mathbf{1 4 6 . 4 3}$ & $\mathbf{6 9 6 . 6 8}$ \\
\hline
\end{tabular}

\section{Usage per service plan -Month3}

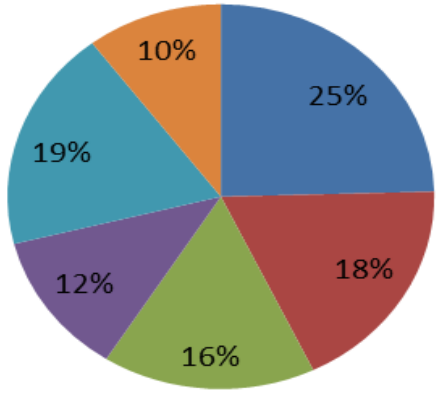

n All_home_Total

- All_SU_Average

all_SOHO Average

- All_Average_SME

all_ME

- All ded_512

Figure 7: Graphical Representation for Third Month

The segmented VLAN network showed improved performance as compared to the original static network.

The results for the initial network without VLANS showed very poor performance during peak hours with the network performance dropping to as low as 30\% for the Home and Single Business users , $25 \%$ for SOHO (Small office network), $20 \%$ for SME,(Small to Medium Enterprise), 10 $\%$ for ME ( Medium Enterprise) but remained at $99 \%$ performance for the dedicated service plans. To improve the network performance we designed and implemented a switched network using layer 3 VLANS on one segment and left the other segment on static routing as the default network. The segmented VLAN network showed improved performance as compared to the original static network. This showed that by creating VLANS we were able to improve network performance by $5 \%[24],[25]$.

However, due to the fact that the number of clients was not fixed in the period under review, it made it difficult to get the conclusive results. The changes in each service plan were inevitable because new clients were registered in each service line, while in some service lines clients closed the service and dropped out.

Table 4 : Data represented over a period of 3 months.

\begin{tabular}{|c|c|c|c|}
\hline Service Plan & Month 1 & Month 2 & Month 3 \\
\hline All_home_Total & 21 & 26 & 25 \\
\hline All_SU_Average & 18 & 21 & 18 \\
\hline All_SOHO Average & 15 & 13 & 16 \\
\hline All_Average_SME & 14 & 17 & 12 \\
\hline All_ME & 16 & 15 & 19 \\
\hline All ded_512 & 16 & 8 & 10 \\
\hline
\end{tabular}

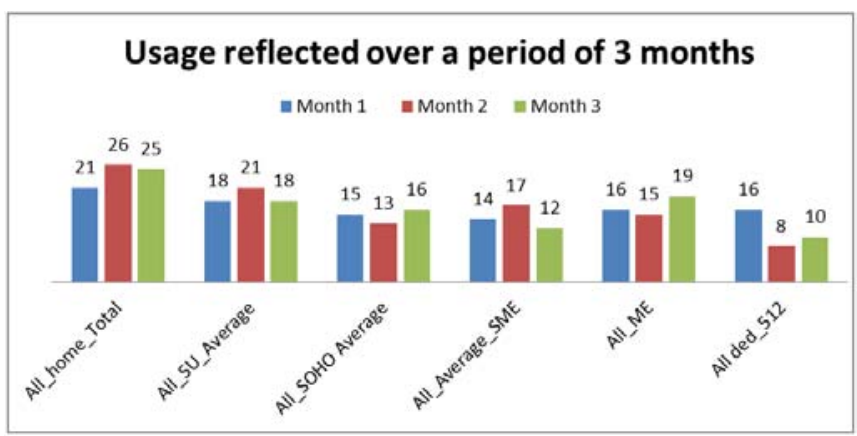

Figure 8: Graphical Representation over 3 Months 


\section{International Journal of Science and Research (IJSR) \\ ISSN (Online): 2319-7064}

Index Copernicus Value (2013): 6.14 | Impact Factor (2014): 5.611

\section{Other recommendations}

As part of the future progress we would like to use network simulator OMNet++ and Dijkstra algorithm to produce desired QoS and efficient bandwidth utilization. To build an MPLS network and re-segment the network. With simulation better results are likely to be obtained.

\section{References}

[1] S. Habeenzu, "Zambia ICT Sector Performance Review 2009/2010," Towar. Evidence-based ICT Policy Regul., vol. Two, 2010.

[2] A. Nafarieh, S. Sivakumar, M. Raza, and W. Robertson, "A review of QoS-based routing mechanisms over shared mesh protected optical infrastructures," Procedia Comput. Sci., vol. 32, no. Iwsron, pp. 1056-1062, 2014.

[3] H. T. Karaoglu, A. Gupta, M. Yuksel, W. Liu, and K. Kar, "Bailout forward contracts for edge-to-edge internet services," Comput. Commun., vol. 36, no. 17-18, pp. 1708-1725, 2013.

[4] A. Abdelkefi, Y. Jiang, B. E. Helvik, G. Biczók, and A. Calu, "Assessing the service quality of an Internet path through end-to-end measurement," Comput. Networks, vol. 70, pp. 30-44, 2014.

[5] M. N. Soorki and H. Rostami, "Label switched protocol routing with guaranteed bandwidth and end to end path delay in MPLS networks," J. Netw. Comput. Appl., vol. 42, pp. 21-38, 2014.

[6] V. Kushwaha and R. Gupta, "Journal of Network and Computer Applications Congestion control for highspeed wired network: A systematic literature review," $J$. Netw. Comput. Appl., vol. 45, pp. 62-78, 2014.

[7] A. B. Bagula, "Hybrid routing in next generation IP networks," Comput. Commun., vol. 29, no. 7, pp. 879892, 2006.

[8] K. Pussep, F. Lehrieder, C. Gross, S. Oechsner, M. Guenther, and S. Meyer, "Cooperative traffic management for video streaming overlays," Comput. Networks, vol. 56, no. 3, pp. 1118-1130, 2012.

[9] X. Yu, G. Xiao, and T. H. Cheng, "Historical data learning based dynamic LSP routing for overlay IP/MPLS over WDM networks," Opt. Fiber Technol., vol. 19, no. 4, pp. 309-318, 2013.

[10] J. Vallet and O. Brun, "Online OSPF weights optimization in IP networks," Comput. NETWORKS, vol. 60, pp. 1-12, 2014.

[11]D. M. Nicol, S. W. Smith, and M. Zhao, "Evaluation of efficient security for BGP route announcements using parallel simulation," Simul. Model. Pract. Theory, vol. 12, no. 3-4 SPEC. ISS., pp. 187-216, 2004.

[12] W. Itani, C. Ghali, R. Bassil, A. Kayssi, and A. Chehab, "ServBGP: BGP-inspired autonomic service routing for multi-provider collaborative architectures in the cloud," Futur. Gener. Comput. Syst., vol. 32, no. 1, pp. 99-117, 2014.

[13] M. P. Howarth, M. Boucadair, P. Flegkas, N. Wang, G. Pavlou, P. Morand, T. Coadic, D. Griffin, A. Asgari, and P. Georgatsos, "End-to-end quality of service provisioning through inter-provider traffic engineering," Comput. Commun., vol. 29, no. 6, pp. 683-702, 2006.
[14] A. Hakiri, A. Gokhale, P. Berthou, D. C. Schmidt, and T. Gayraud, "Software-Defined Networking: Challenges and research opportunities for Future Internet," Comput. Networks, vol. 75, pp. 453-471, 2014.

[15] J. Calzada and F. Martínez-santos, "Broadband prices in the European Union: Competition and commercial strategies," Inf. Econ. Policy, vol. 27, pp. 24-38, 2014.

[16]R. Rajabiun and C. A. Middleton, "Multilevel governance and broadband infrastructure development: Evidence from Canada \$," Telecomm. Policy, vol. 37, no. 9, pp. 702-714, 2013.

[17]F. Belloc, A. Nicita, and M. A. Rossi, "Whither policy design for broadband penetration? Evidence from 30 OECD countries," Telecomm. Policy, vol. 36, no. 5, pp. 382-398, 2012.

[18] T. Turk and P. Trkman, “Technological Forecasting \& Social Change Bass model estimates for broadband diffusion in European countries," vol. 79, pp. 85-96, 2012.

[19] S. Jay, P. Anell, T. Plückebaum, G. Kulenkampff, and S. Marcus, "Next Generation Bitstream Access," no. November, 2007.

[20]I. F. Akyildiz, A. Lee, P. Wang, M. Luo, and W. Chou, "A roadmap for traffic engineering in software defined networks," Comput. Networks, vol. 71, pp. 1-30, 2014.

[21]M. Chiesa, G. Lospoto, M. Rimondini, and G. Di Battista, "Intra-domain routing with pathlets," Comput. Commun., vol. 46, pp. 76-86, 2014.

[22] E. Baralis, A. Bianco, T. Cerquitelli, L. Chiaraviglio, and M. Mellia, "NetCluster: A clustering-based framework to analyze internet passive measurements data," Comput. Networks, vol. 57, no. 17, pp. 33003315, 2013.

[23]Z. Dong, R. D. W. Perera, R. Chandramouli, and K. P. Subbalakshmi, "Network measurement based modeling and optimization for IP geolocation," Comput. Networks, vol. 56, no. 1, pp. 85-98, 2012.

[24] C. Used, C. I. Routing, S. Instructions, T. Procedure, R. Information, R. Cisco, and S. Community, "Configure InterVLAN Routing Task."

[25] M. Adler, R. K. Sitaraman, and H. Venkataramani, "Algorithms for optimizing the bandwidth cost of content delivery," Comput. Networks, vol. 55, no. 18, pp. 4007-4020, 2011.

\section{Author Profile}

Tute $\mathbf{L}$ is currently working for ZAMNET Communication Systems Limited, as the Head of Technical Department. He obtained his Bachelor of Science at the University of Zambia in 2000, and currently pursuing a MEng in ICT at the University of Zambia. His research interests include Routing/Switching and Optimization of Internet Service Provider networks and information security.

Phiri $\mathbf{J}$ is currently working for the University of Zambia in the Department of Computer Science. He is the current Chair of the Department of Computer Science at the University of Zambia. He obtained his Bachelor of Computer Science at the University of Zambia in 2004, an MSc in Computer Science at the University of the Western Cape in South Africa in 2007 and a PhD in Computer Science at Harbin Institute of Technology in China in 2012. His research interest includes Identity Management, Data Mining, 
applied artificial Intelligence technologies and Information Security.

Phiri A is currently working for the Zambia Airports Corporation Limited, as the Head of Projects. He obtained his Bachelor of Engineering at the University of Zambia in 2005, and currently pursuing a MEng in ICT Policy, Regulation and Management at the University of Zambia. He is a Fellow Member of the Engineering Institution of Zambia. He is also a member of the IEEE and ACM. His research interests include telecommunication Policy and Regulation, Food Supply Chain Management and computer communication systems. 